\title{
Trend Analyses Revision and Global Monthly Temperature Innovative Multi-Duration Analysis
}

\author{
Abdullah M. Mohorji ${ }^{1}$ Zekâi Şen $^{2} \cdot$ Mansour Almazroui $^{2}$
}

Received: 15 February 2017/Revised: 1 August 2017/Accepted: 5 August 2017/Published online: 22 August 2017

(C) The Author(s) 2017. This article is an open access publication

\begin{abstract}
Background Climate change evidence has been documented by different authors based on the long years' monthly temperature measurements since long back. In the literature, annual-mean and 5-year moving average time series of global mean land-ocean temperature index and meteorological station data of global annual-mean surface air temperature changes are presented with a base period including some parts as uncertainty estimates. This paper provides an innovative method for refined calculation of global warming calculation.

Purpose The innovative multi-duration trend analysis application to the global monthly temperature data for identification of monthly temperature variability leads to temperature increase identifications in an innovative manner. The purpose is to present a detailed and refined innovative trend application methodology for global temperature increment calculation.

Methods After the general revision of non-parametric and parametric trend methodology explanations, the innovative trend template (ITT) analysis application is presented by considering two-half and multi-duration trend possibilities in the global monthly temperature records.

Results The ITT methodology also presents various features of the global temperature increments during the whole record duration on monthly basis leading to a set of
\end{abstract}

Zekâi Şen

zsen@kau.edu.sa

1 Department of Civil Engineering, Faculty of Engineering, King Abdulaziz University, Jeddah, Saudi Arabia

2 Department of Meteorology, Center of Excellence for Climate Change Research, King Abdulaziz University, PO Box 80234, Jeddah 21589, Saudi Arabia verbal interpretations and numerical values for each month including "Low" (minimum), "High" (maximum), and "Medium" (moderate) temperature amounts. It is proven that, on average, there is $0.9{ }^{\circ} \mathrm{C}$ and $1.78{ }^{\circ} \mathrm{C}$ monthly temperature increments for "Low" and "High" temperatures, respectively, in addition to average incremental temperature of $1.33{ }^{\circ} \mathrm{C}$.

Conclusion The innovative trend template (ITT) methodology is explained briefly and applied to global monthly temperature records from 1881 to 2013. This new methodology provides information about "Low" (minimum) and "High" (maximum) temperature records in addition to the "Medium" transitional temperatures. First, two-half and then multi-period innovative trend analysis implementations are explained graphically, verbally, and numerically. Finally, the ITTs application indicated that the warming at global scale is at about $0.75{ }^{\circ} \mathrm{C}$, which was determined by some other approach as $0.76{ }^{\circ} \mathrm{C} \pm 0.19{ }^{\circ} \mathrm{C}$ (IPCC, 2007).

Keywords Analysis - Climate change - Global temperature Innovative $\cdot$ Multi-duration template $\cdot$ Trend

\section{Introduction}

Climate change poses particular risks for human nutrition sources such as water resources, agriculture, and food products. Apart from the natural internal and external forcing processes such as volcanic eruptions and solar cycles, persistent anthropogenic impacts play an undesirable role in the composition of the atmosphere or land use. United Nation Framework Conservation on Climate Change (UNFCCC) defines climate change as "a change of climate which is attributed directly or indirectly to human 
activity that alters the composition of the global atmosphere and which is in addition to nature climate variability observed over comparable time periods".

Over the past three centuries, human activities have impacted on the earth system behavior at the global scale, more so than at any other time. The cumulative impacts of anthropogenic activities are felt on the structure and functioning of the Earth systems. These impacts have profound effects on the lithosphere, atmosphere, coastal areas, and the cryosphere. Apart from the perturbative effect of the carbon cycle, fossil fuel burning has been the most prominent factor for climate change over the past 2 to 3 decades. For the past several decades, measurement records of global temperature, carbon dioxide, nitrate, sulfur, methane, and other chemical elements have been kept. Hence, it is now possible to treat the numerical values in the form of time series by different methodologies so as to explore internal systematic structures such as trends, which provide scientific information for better modeling, prediction, and control mechanism of the phenomenon concerned. Such scientific information provides basic verbal and numerical data for local and central administrators nationally and internationally.

It is by now well established in the literature that climate change has intensified the hydrological cycle (IPCC 2007, 2013). The intensification generates hydrological variables such as increasing numbers of increasingly severe flood and drought occurrences. It is, therefore, necessary to not only have trend identification techniques for the whole record duration, but also, more significantly, to compare last year's portion with earlier records for a better understanding of the general tendency features. During the last 2-3 decades, there has been a proliferation of trend studies, using a diversity of hydro-climatological variables.

There are numerous classical trend application studies in the literature for various regions of the world, but most of them provide monotonic trends over the whole record period. In the past, many environmental planning, management, and operation studies assumed implicitly that the time series (temperature, precipitation, and stream-flow records) are stationary (Maass et al. 1962; Milly et al. 2008). However, the stationarity assumption is no longer valid due to anthropogenic effects in the atmospheric and hydrologic environments (IPCC 2007, 2013). By now, numerous studies have demonstrated that the stationarity principle is dead because of substantial variabilities due to climate change impacts on earth systems. Changes in the means of earth system time series and in their extreme values may imply trend existence, which must be identified and separated from the main series so as to render it into a stationary state. For the past 3 decades, earth systems and their direct impacts on natural environment have been searched through trend analysis, especially by employing Mann-Kendall (MK) analysis (Mann 1945; Kendall 1975) coupled with the Sen (1968) trend slope calculation methodology. The climate change triggered trend analysis applications at an unprecedented rate, and consequently, many researchers in environment and atmospheric research domains applied the MK methodology for possible trend search (Hirsch et al. 1982; van Belle and Hughes 1984; Hirsch and Slack 1984; Cailas et al. 1986; Hipel et al. 1988; Demaree and Nicolis 1990; Yu et al. 1993; Gan 1998; Taylor and Loftis 1989; Lins and Slack 1999; Kalra et al. 2008).

Efficient, effective, and optimum management of earth systems not only requires monotonic trend identifications, but also requires inspection of whether or not the "low", "medium", and "high" values have separate trends. In general, a monotonic trend has a gradual change over the whole record period and it is expected to continue in the future. However, for "low", "medium", and "high" value trend searches, the periods are comparatively shorter than the record period. Zhang et al. (2010) suggested that the literature so far has devoted very limited attention to the characterization of trend pattern. The literature is full of local, regional, and national trend studies much more than the innovative methodological techniques to improve some drawbacks due to restrictive assumptions in the classical trend test techniques. Although Visser et al. (2015) have reviewed many papers that attempt to develop many new statistical methods to describe the variability of trends, but they did not include the innovative trend template (ITT) analysis methodology (Şen 2012) in their review.

The main purpose of this paper is to show the applicability of the innovative trend analysis methodology without restrictive assumptions. The suggested methodology helps to identify not only possible trends in "low", "medium", and "high" time series values separately, but also the trend identification and determination over a succession of multidurations. A detailed discussion is presented for annual and monthly global temperature records from innovative trend template point of view, which provides meaningful information different from classical methodologies.

\section{General Review of Trend Methodologies}

In general, trend methodologies can be classified into two broad branches: non-parametric and parametric approaches. The former group of alternatives is robust and probability distribution function (PDF) free, based on ascending order ranks. The transformation of natural record sequence to ascending order causes information losses; therefore, the results are rather conservative in the sense that null hypothesis of no-trend existence is very unlikely. These methodologies must be used with care of the basic assumptions. Direct non-parametric test applications are 
possible in cases of ordinal data availability, which do not give way to calculate basic statistical parameters such as the arithmetic average and standard deviations that are needed in parametric methodology implementations. On the other hand, the data PDF of small sample shows tendency to deviate from a normal (Gaussian) PDF, which is another requirement for non-parametric trend applications.

\subsection{Non-Parametric Trend Tests}

In almost all trend analyses, the first step is to identify possible trend existence by non-parametric methods, followed by the application of parametric trend determination as a linear equation. Non-parametric trend analysis techniques also provide the trend slope, but parametric approaches such as the regression analysis are very helpful for visual conceptual grasp and making meaningful conclusion. However, after non-parametric trend slope calculation, the generation of the straight-line trend is rather different from the parametric regression trend methodology. In the non-parametric approaches, provided that the trend slope is calculated in some way, the straight-line trend is assumed to pass from the centroid of a given time series. The centroid is a point with the arithmetic averages of a given time series variable on the vertical axis with the half-record duration on the horizontal axis (Fig. 1).

The classical Mann-Kendall non-parametric trend tests are based on the median slope value as suggested by Sen (1968), which is not sensitive to outliers. If time series, $X_{i}$ ( $i=1,2, \ldots, n)$ with sample length, $n$, is given, then there will be $n(n-1) / 2$ possible pairs of time points $(i, j)$ in which $i>j$. Hence, a pairwise slope, $b_{i j}$, can be obtained simply as follows:

$b_{i, j}=\frac{X_{j}-X_{k}}{j-k} \quad($ for $j>k ; i=1,2, \ldots, n)$.

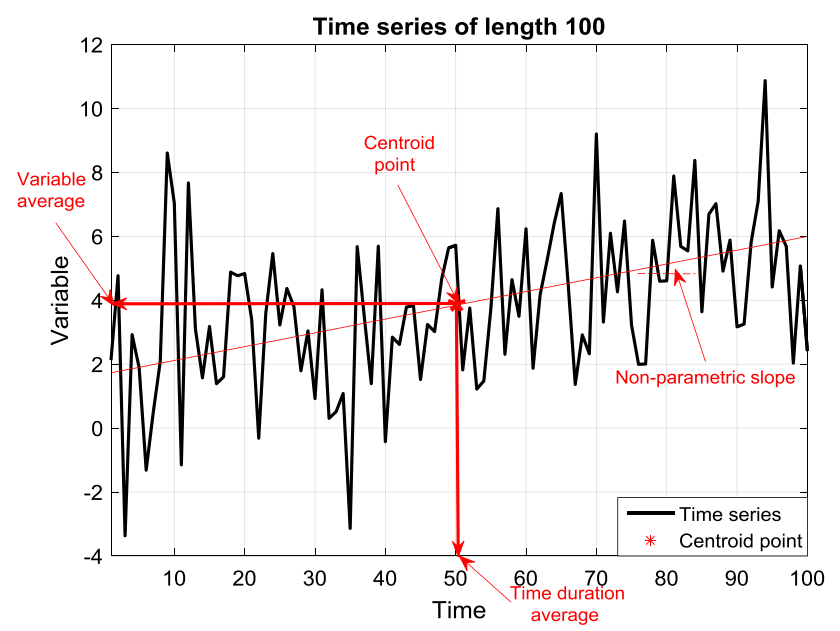

Fig. 1 Non-parametric trend line construction
The overall Sen slope, $S_{\mathrm{s}}$, of the trend is adapted as the median value of these slopes. If the number of positive slopes is almost equal to the negative slopes, there is notrend in practice. Otherwise, there is an increasing (decreasing) trend provided that the number of positive (negative) slopes is more than negative (positive) slopes. More formally, a two-tailed significance test can be obtained concerning the slope value by the non-parametric technique based on the normal (Gaussian) PDF. It is possible to calculate the confidence limits as (Şen 2017a) follows:

$\mathrm{CL}(\alpha)=\mp Z_{i-\alpha / 2} \sqrt{\operatorname{Var}(S)}$.

The MK test requires normal (Gaussian) PDF of a serially independent time series. Most trend-detection studies attached with the MK trend test assume that records are serially independent, even though hydro-climatological time series have statistically significant serial correlations (Yue and Wang 2004). For instance, von Storch (1995) documented that the existence of positive serial correlation increases the probability that the MK test detects trends when no-trend exists. To overcome MK test restrictive assumptions, Yue et al. (2002) explored the influence of pre-whitening on the pre-whitened series only in the cases that time series had an upward trend in a lag-one autoregressive stochastic process. Pre-whitening is a procedure for reduction of serial correlation within a given time series by adding white noise (serially independent) series to the original records. Unfortunately, pre-whitening has been applied to temperature and stream-flow trend analyses prior to MK test trend identification without caring for independence structure (Zhang et al. 2001; Hamilton et al. 2001; Burn and Hag Elnur 2002). Yue and Wang (2004) have extended similar pre-whitening treatment through extensive Monte Carlo simulation studies prior to the application of the MK trend procedure to various

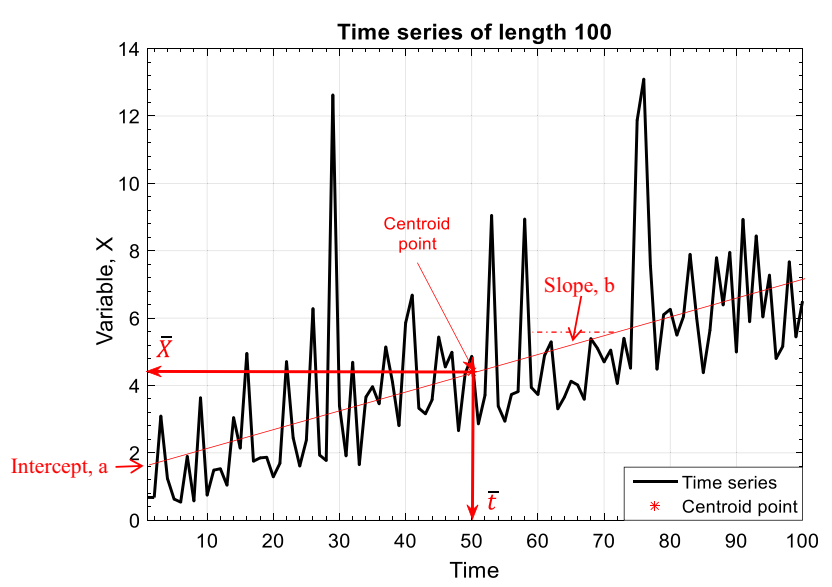

Fig. 2 Parametric trend line constructions 
combinations of the first-order autoregressive process with increasing and decreasing trends. They have concluded that trend existence in a time series depends on the sample size, serial correlation magnitude, and trend slope amount.

\subsection{Parametric Trend Tests}

Parametric statistics should be used with sample sizes of more than 30 , provided that the data conform to normal (Gaussian) PDF. It is important to remember that all means of transformations should be applied to the data at hand in an attempt to normalize the data before parametric methods are abandoned. The mathematical forms of the trends are either a straight-line or low-order polynomial. The graphical representation of a time series with an increasing trend component is shown in Fig. 2.

Parametric regression of variable $X$ on time, $t$, is expressed as a monotonic straight-line with intercept, $a$, slope, $b$, and random variable, $\varepsilon$, mathematically as follows:

$X=a+b t+\varepsilon$.

In case of no-trend, the null hypothesis is that the slope is equal to zero $(b=0)$. The classical regression methodology has to pass through the centroid, i.e., arithmetic average values of the two variables, $(\bar{X}, \bar{t})$, which leads to the following:

$\bar{X}=a+b \bar{t}$.

Another expression can be found after multiplying both sides of Eq. (3) by the independent $t$ variable and then taking the arithmetic averages of both sides, giving

$\overline{X t}=a \bar{t}+b \overline{t^{2}}$.

The simultaneous solution of the last two equations yields $a$ and $b$ parameter values. The parametric regression has stronger assumptions than the classical Mann-Kendall trend test, because the residuals, i.e., $\varepsilon$ values, should accord with normal (Gaussian) PDF. In addition, homoscedasticity (standard deviation constancy) must be checked. In cases of non-linearity, the variables can be subjected to a suitable transformation such as the logarithmic, square root, cubic, or any other type. After the confirmation of all these rather restrictive assumptions, $\mathrm{t}$ tests can be applied on $b$ to determine if it is significantly different from zero, which implies the existence of a trend (Şen 2017).

\section{Monthly World Temperature Time Series}

The monthly global temperature data are obtained from National Center for Atmospheric Research (NCAR) center from 1881 to 2013. Annual global land-ocean temperature

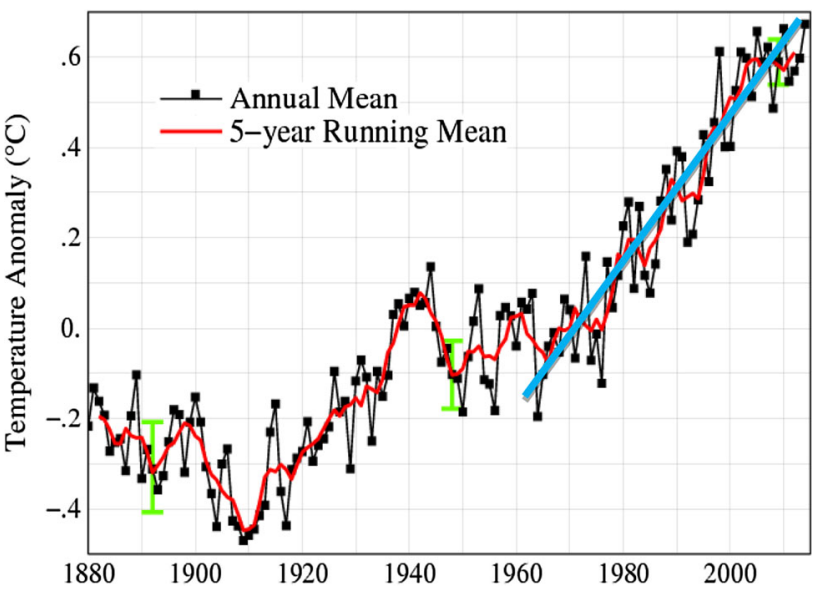

Fig. 3 Global annual mean and 5-year running mean temperature series

values are shown in the form of time series in Fig. 3, where a linearly increasing trend appears after the 1970s, which shows the reflection of anthropogenic effects on the atmospheric composition as a result of greenhouse gases emissions.

At the first glance, the global temperature records since 1881 indicate warming along several decades with interannual variability, which is a very significant concept in the climate change studies, because such variabilities may offset the possible climate change trends over short durations. Besides, any climate change study trends or weighting averages considers the arithmetic average levels without any consideration of departures from these trends.

Annual mean and 5-year running mean (moving average) time series plot of global mean land-ocean temperature index is presented in Fig. 4 with the base period 1951-1980 with some parts as uncertainty estimates (Hansen et al. 2010, Fig. 9a).

Figure 5 indicates annual and 5-year running mean values time series traditional analyses using only meteorological station data of global annual mean air temperature

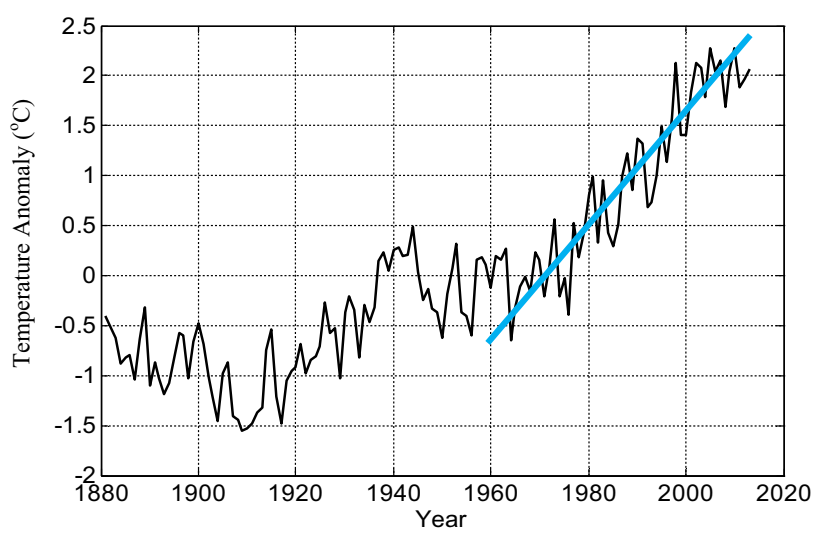

Fig. 4 Global annual temperature anomalies 


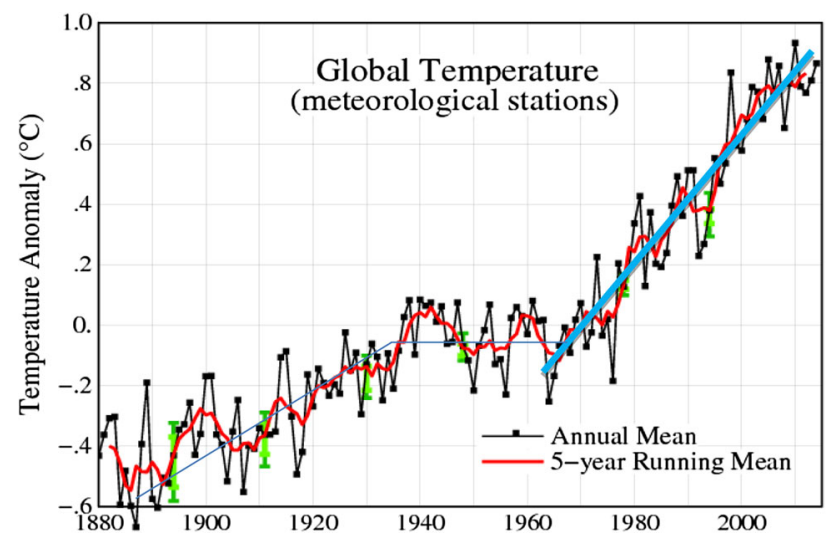

Fig. 5 Global annual mean temperature time series with recent trend

change, again with the base period 1951-1980. This graph is derived from the meteorological station network (Hansen et al. 2001).

In all three figures, temperature increase anomaly starts around the 1970s in an unprecedented manner and still the same trend goes on in a linear form. In these figures, the linear trends along the recent portions are fixed by eye to show the significance of recent temperature trends.

\section{Innovative Trend Template Methodology}

Trend methodologies are varied in open literature each with a set of restrictive assumptions. As explained earlier, the MK trend test is the most well-known and frequently used among them for trend depiction, with the slope calculation through the median slope method. These nonparametric approaches are used in many earth system studies for monotonic trend detection in a given time series data.

In the simplest form of the innovative trend template (ITT) approach, the given time series is divided first into

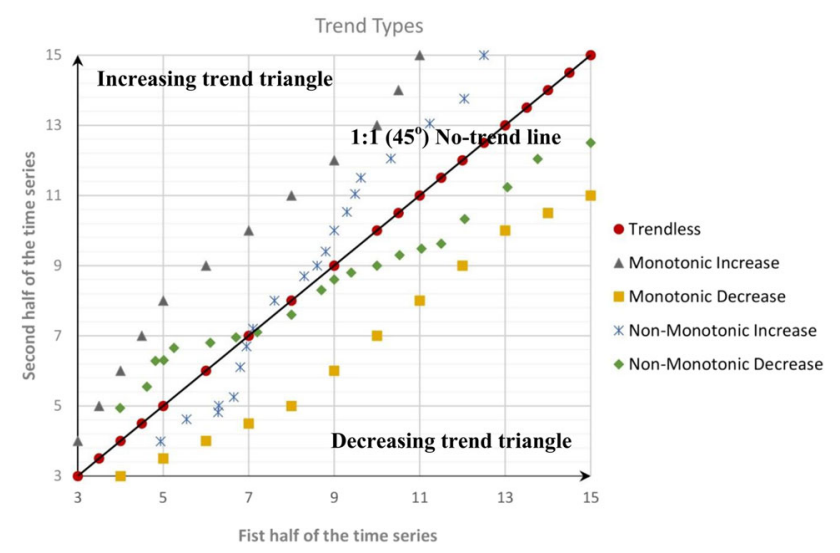

Fig. 6 Illustration of decreasing, increasing, and trendless regions (Dabanli et al. 2016) two equal sub-segments, then each one is ordered ascendingly, and finally, they are plotted against each other to obtain the scatter of points, such that the "low" ("medium" and "high") values of the first half appears against the "low" ("medium" and "high") values of the second half. If the time series is composed of terms $X_{1}, X_{2}, X_{3}, \ldots$, $X_{n}$, the first half series $\left(X_{i}: i=1,2, \ldots, n / 2\right)$ is shown on the horizontal axis against the second half $\left(X_{j}: j=n / 2+1, n /\right.$ $2+2, \ldots, n)$ on the vertical axis of the Cartesian coordinate system leading to a scatter diagram similar to the one in Fig. 6.

For simple comparison purposes, the scale and variation domain range of both axes should preferably be equal. In case the scatter points fall exactly or approximately around the vicinity of $1: 1\left(45^{\circ}\right)$ straight line, there is in practice no significant trend in earth system time series (trendless time series). On the contrary, either an increasing or decreasing trend exists within the time series. The $1: 1\left(45^{\circ}\right)$ straight line divides the template into two equal triangular areas. The upper (lower) triangular areas are for increasing (decreasing) trend components (Şen 2012). It is possible to categorize the variation domain of each half series into a set of clusters as "low", "medium", and "high". The ITT scatter diagram provides first visual inspection about the trend types in each category.

The range of each cluster can be decided either according to expert opinion, or quantitatively by dividing the domain of variation into equal parts. Similar concepts can be extended for any duration within the given time series. For instance, if duration, $d(d<n)$, is adapted, then there will be $n / d$ integer number of successive durations within the time series. Consideration of two successive durations similar to aforementioned two-half case yields ITTs, and hence, a multi-duration trend interpretation can be achieved.

\section{Application}

In the application of the ITT methodology, in general, there are two potential approaches: division of the original time series into two equal sub-segments, which is explained in the previous section, or a multiple division into equal duration multi-duration sections.

\subsection{Two-Part ITT}

The application of the ITT methodology to monthly global temperature records provides graphs that present scientific verbal and numerical information that can be extracted visually (Fig. 7). Although there are similar graphs for each month, for the sake of space saving, only one month is 
Fig. 7 ITTs for global monthly temperatures
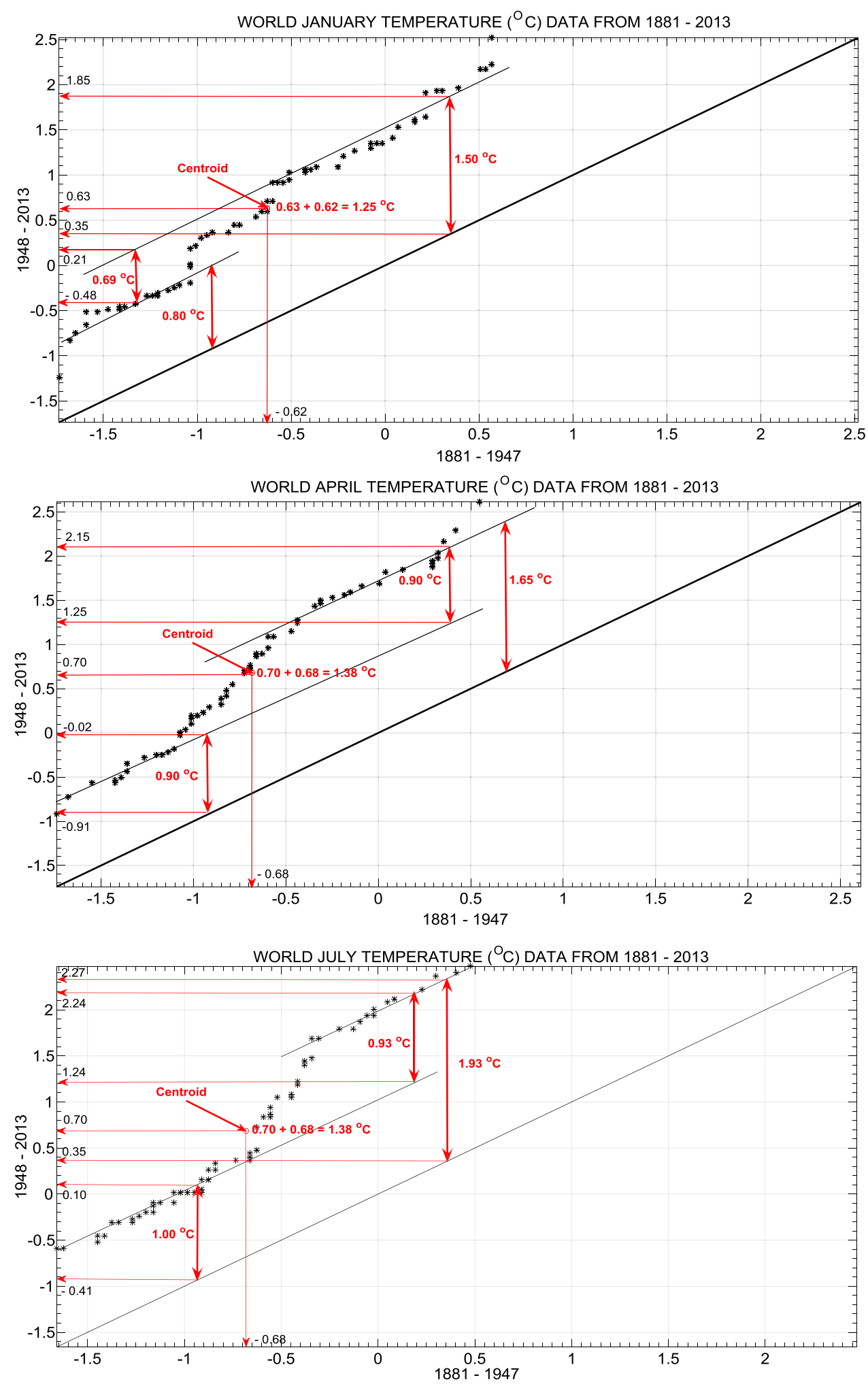

presented for each season in this paper. First of all, it is helpful for subsequent numerical interpretations to extract a set of possible verbal information among which are the following points.
1. Throughout the graphs, it is obvious that, after 1947, there are increasing trends both in the minimum (low) and maximum (high) monthly temperatures. This point is documented by the fact that all the scatter points in 
Fig. 7 continued

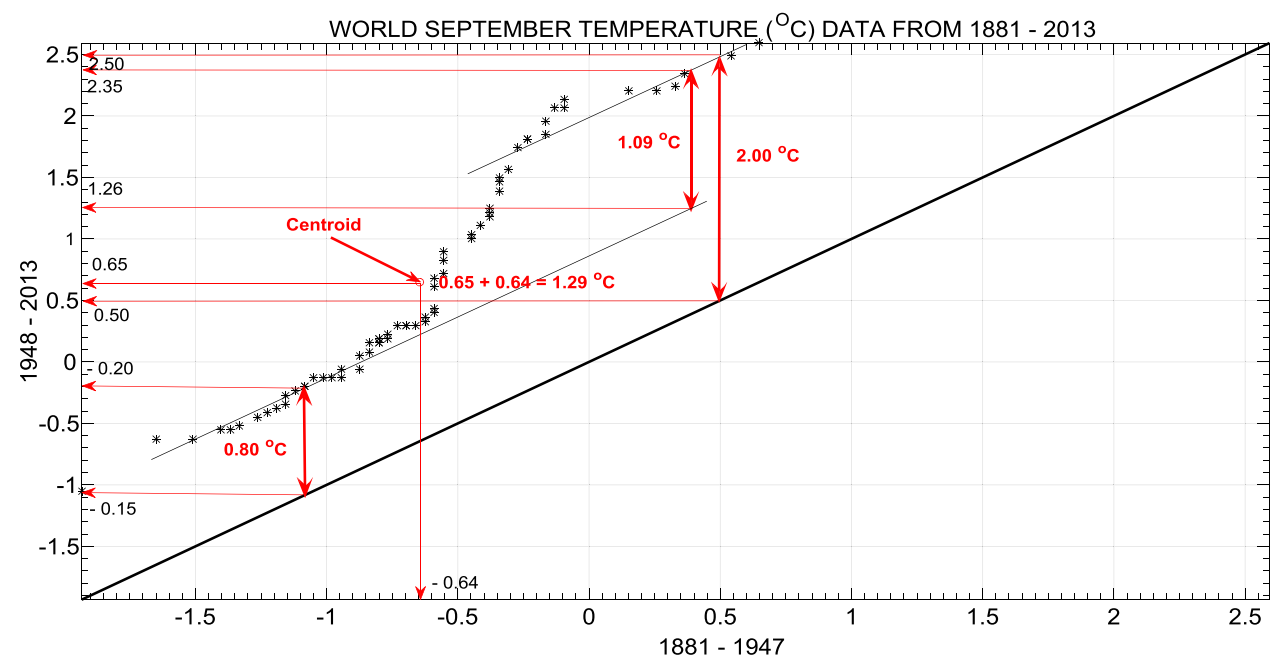

the graphs fall within the upper triangular area above the $1: 1\left(45^{\circ}\right)$ straight line that represents the case of notrend within the record time series; "low" and "high" temperature value scatter points are clustered along parallel straight lines to the $1: 1\left(45^{\circ}\right)$ line, and therefore, there are no significant variabilities in the deviations from the arithmetic averages of "low" and "high" temperature clusters. This is tantamount to saying that the standard deviations along these two classes are constant (homoscedastic).

2. Global temperature increment in "high"-temperature values is more than the low-temperature records, because the scatter points are far away from the $1: 1$ $\left(45^{\circ}\right)$ no-trend straight line, "Medium" temperature values fall within the transition zone from the "low"to "high"-temperature values, and their scatter points do not fall around a parallel line to the $1: 1\left(45^{\circ}\right)$ straight line, which shows the implication that the "Medium" temperatures have variability, i.e., heteroscedasticity (variation in the standard deviation) "Medium"-temperature increment is more than the low temperatures but less than the high-temperature records. The centroid point as the arithmetic averages of first and second halves falls within the "medium" temperature values range.

On the other hand, the numerical evaluation of the same ITTs provides another set of information that is useable in any numerical modeling. For this purpose, all the numerical values shown in Fig. 7 are presented in Table 1.

On the basis of comparison of the late half of the global temperature records with the early half, one can observe that there is always an increasing trend after 1947, which is obvious from the numerical values at the last row of Table 1. The last column of this table indicates that "low" and "high" average monthly temperature increments with respect to the no-trend line are 0.90 and $1.78{ }^{\circ} \mathrm{C}$, respectively, with "moderate" increment of $1.34{ }^{\circ} \mathrm{C}$. Hence, the average variation domain between minimum and maximum temperatures is $1.78-0.90=88^{\circ} \mathrm{C}$.

Cohn and Lins (2005) and Koutsoyiannis and Montanari (2007) stated that it is possible to think about the assessment of trend statistical significance test for this method. This approach is graphically based which is a preferable way to address the issue of trend, in that it avoids the pitfalls of attempting to ascribe statistical significance to a poorly understood system. One can argue strongly that the concept of statistical significance is meaningless when discussing poorly understood systems, because significance depends critically on the null hypothesis, which in turn reflects subjective notions about what one expects to see. Therefore, unless one can specify the null hypothesis correctly, the resulting estimate of significance will be wrong. In this paper, their advice is adapted, because the graphical approach is a preferable way to address the issue of trend in that it avoids the pitfalls of attempting to ascribe statistical significance to a poorly understood system. Furthermore, a statistical analysis method has already been suggested by Şen (2017b).

To visualize and make further meaningful interpretations, the "low"- and "high"-temperature values are drawn in Fig. 8.

It is possible to extract from this figure that on monthly basis, there has been $0.50{ }^{\circ} \mathrm{C}$ temperature changes on the average for the "high" global temperature records. However, among the monthly "low" global temperature values, it is $0.25{ }^{\circ} \mathrm{C}$ annually. These two statements indicate that the climate change reflection in the "high" maximum temperatures is twice that of the "low", minimum monthly records. Another conclusion is that during the last decades, the warming at global scale is about $0.50+0.25$ $=0.75{ }^{\circ} \mathrm{C}$ and this is very close to IPCC (2007) report finding, which states without any distinction between the 
Table 1 Numerical value sets of Fig. 7

\begin{tabular}{|c|c|c|c|c|c|c|c|c|c|c|c|c|c|}
\hline \multirow[t]{2}{*}{ Trend features } & \multicolumn{12}{|c|}{ Monthly temperature $\left({ }^{\circ} \mathrm{C}\right)$ features } & \multirow{2}{*}{$\begin{array}{l}\text { Mean } \\
\left({ }^{\circ} \mathrm{C}\right)\end{array}$} \\
\hline & Jan. & Feb. & Mar. & Apr. & May & Jun. & Jul. & Aug. & Sep. & Oct. & Nov. & Dec. & \\
\hline "Low"-temperature trend & 0.80 & 1.00 & 0.75 & 0.90 & 1.00 & 1.00 & 1.00 & 0.75 & 0.80 & 0.90 & 0.90 & 0.95 & 0.90 \\
\hline "High"-temperature trend & 1.50 & 1.50 & 1.70 & 1.65 & 1.90 & 2.00 & 1.90 & 2.00 & 2.00 & 1.75 & 1.85 & 1.60 & 1.78 \\
\hline Mean trend & 1.15 & 1.25 & 1.23 & 1.27 & 1.45 & 1.50 & 1.45 & 1.38 & 1.40 & 1.32 & 1.38 & 1.28 & 1.34 \\
\hline \multicolumn{14}{|l|}{ Mean } \\
\hline $1881-1947$ & -0.62 & -0.65 & -0.65 & -0.68 & -0.770 & -0.72 & -0.68 & -0.68 & -0.64 & -0.62 & -0.61 & -0.66 & -0.67 \\
\hline 1948-2013 & 0.63 & 0.70 & 0.65 & 0.70 & 0.74 & 0.75 & 0.70 & 0.54 & 0.65 & 0.60 & 0.63 & 0.68 & 0.66 \\
\hline
\end{tabular}

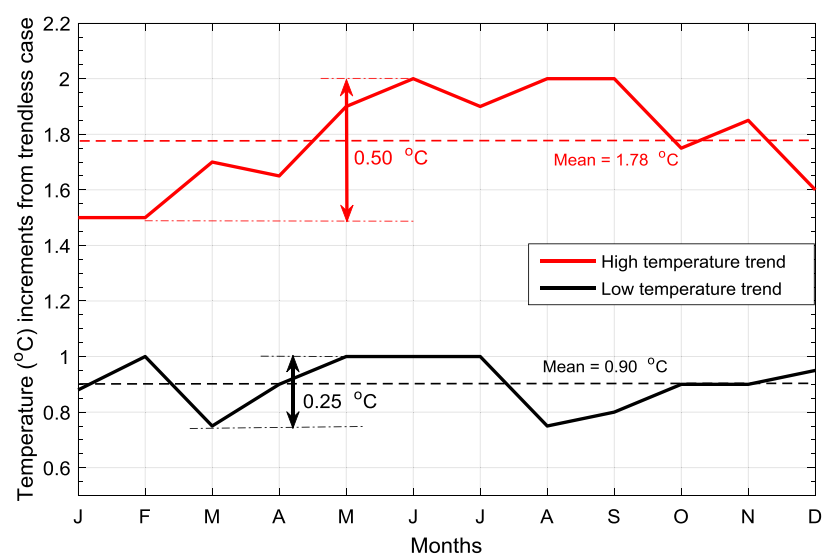

Fig. 8 Monthly "low" and "high" global monthly temperature trends

minimum and maximum temperatures that the total temperature increase from 1850-1899 to 2001-2005 is $0.76 \pm 0.19{ }^{\circ} \mathrm{C}$.

\subsection{Multi-Duration Trend Analysis}

It is possible to widen the two-half trend identification to any desired duration trend identification for the global temperature time series. Figure 9 represents the ITTs for a set of various decades based on the first basic decade length. In this paper, 10-, 20-, 30-, 40-, and 50-year durations are considered as the multiple-duration trend identifications. Again, the first impressions and verbal interpretations about these graphs can be summarized as follows:

1. In Fig. 9a, for each decade based on the first decade of 1881-1890, the scatter points of subsequent decades on the ITT indicate that although 1891-1900 decade is almost trend free, because its scatter points are the closest to the $1: 1\left(45^{\circ}\right)$ straight-line, but subsequent two decades, namely, 1901-1910 and 1911-1920, had decreasing trends in "low"-, "medium"-, and "high"temperature values, because all of the points are below the no-trend line.

2. Starting with the 1921-1930 decade, global temperatures indicate steadily increasing temperature trends of $2{ }^{\circ} \mathrm{C}$ in 2001-2010 duration, which is very obvious in Fig. 9a. After 1981 especially, each decade exposes a distinctive difference between the "low"- and "high"temperature values, because the scatter of points for these decades does not fall on a single straight line that is parallel to $1: 1\left(45^{\circ}\right)$ no-trend line.

3. As for the scatter of points on the basis of the twodecade periods in Fig. 9b, it is obvious that 1901-1920 period has slightly decreasing trend, but in all periods except 1981-2000, there are almost parallel straight lines to the $1: 1\left(45^{\circ}\right)$ straight line. This means that, as in the previous item, different climate change patterns started to appear between the "low"- and "high"temperature values after 1981 .

4. In Fig. 9c, the ITT is constructed for a basis of 3 decades (1881-1910), and then, the subsequent triple decade temperature scatter points' behavior shows that 1911-1940 and 1941-1970 periods have rather stable and continuously increasing temperature without any distinction between the "low" and "high" global temperatures, because the straight lines to these periods are almost parallel to the $1: 1\left(45^{\circ}\right)$ no-trend line, but after 1971, there is no parallelism again indicating the different behaviors between the "low"and "high"-temperature changes.

5. Four-decade period based on 1881-1920 ITT is presented in Fig. 9d, where there are two subsequent 4-decade periods, i.e., 1921-1960 and 1961-2000. On considering such long durations (more than 30-year meteorology base line), one can observe that there are only increasing temperature trends, but the behavior during the last 4 decades, i.e., 1961-2000, again shows that there is not a single straight line for all the scatter points, which implies as the difference between the 


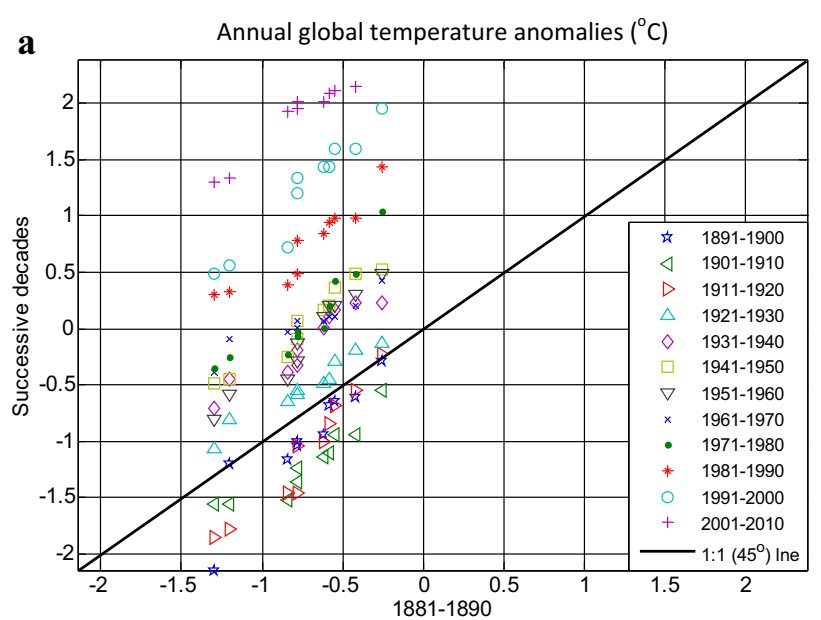

b Annual global temperature anomalies $\left({ }^{\circ} \mathrm{C}\right)$
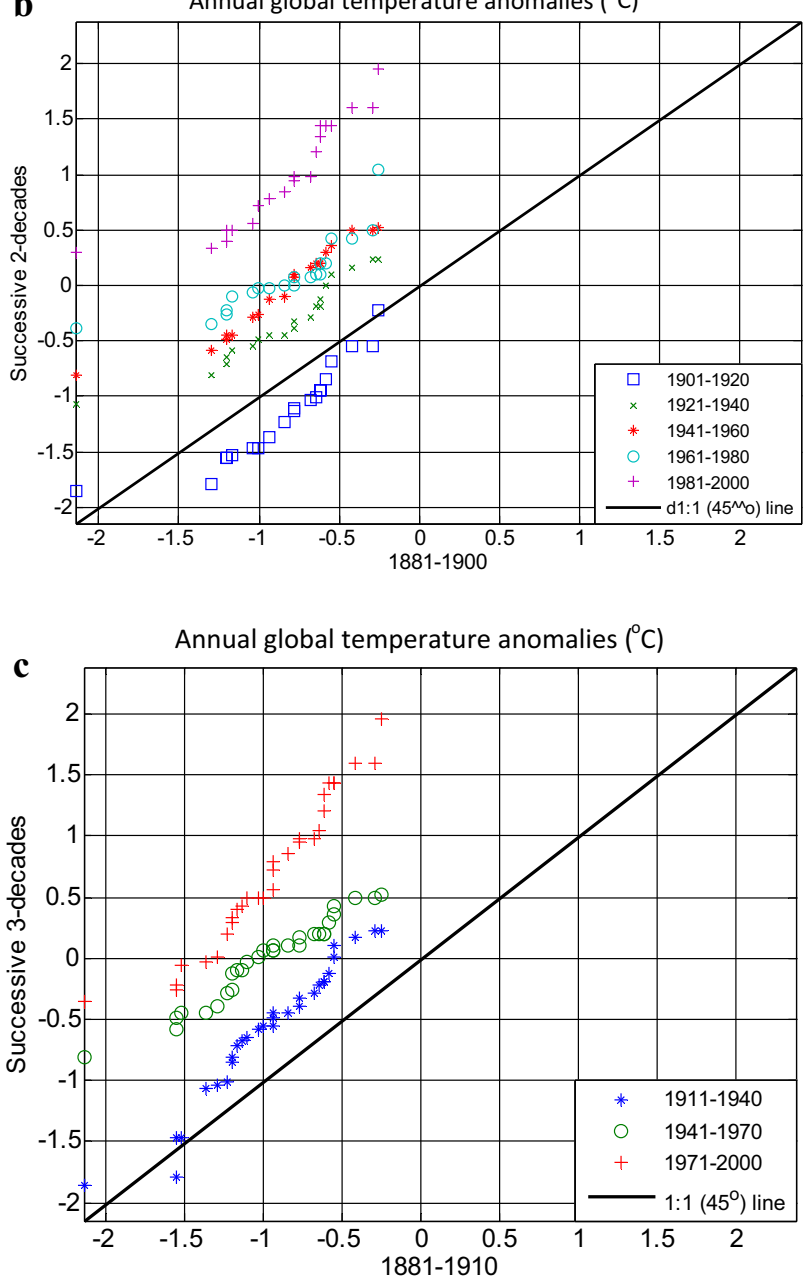

Fig. 9 ITT for 10-, 20-, 30-, 40-, and 50-year durations

"low"- and "high"-temperature variations for the previous case.

6. Finally, in Fig. 9e, 5-decade basic period (1881-1930)based ITT graph is given with only one replicate within
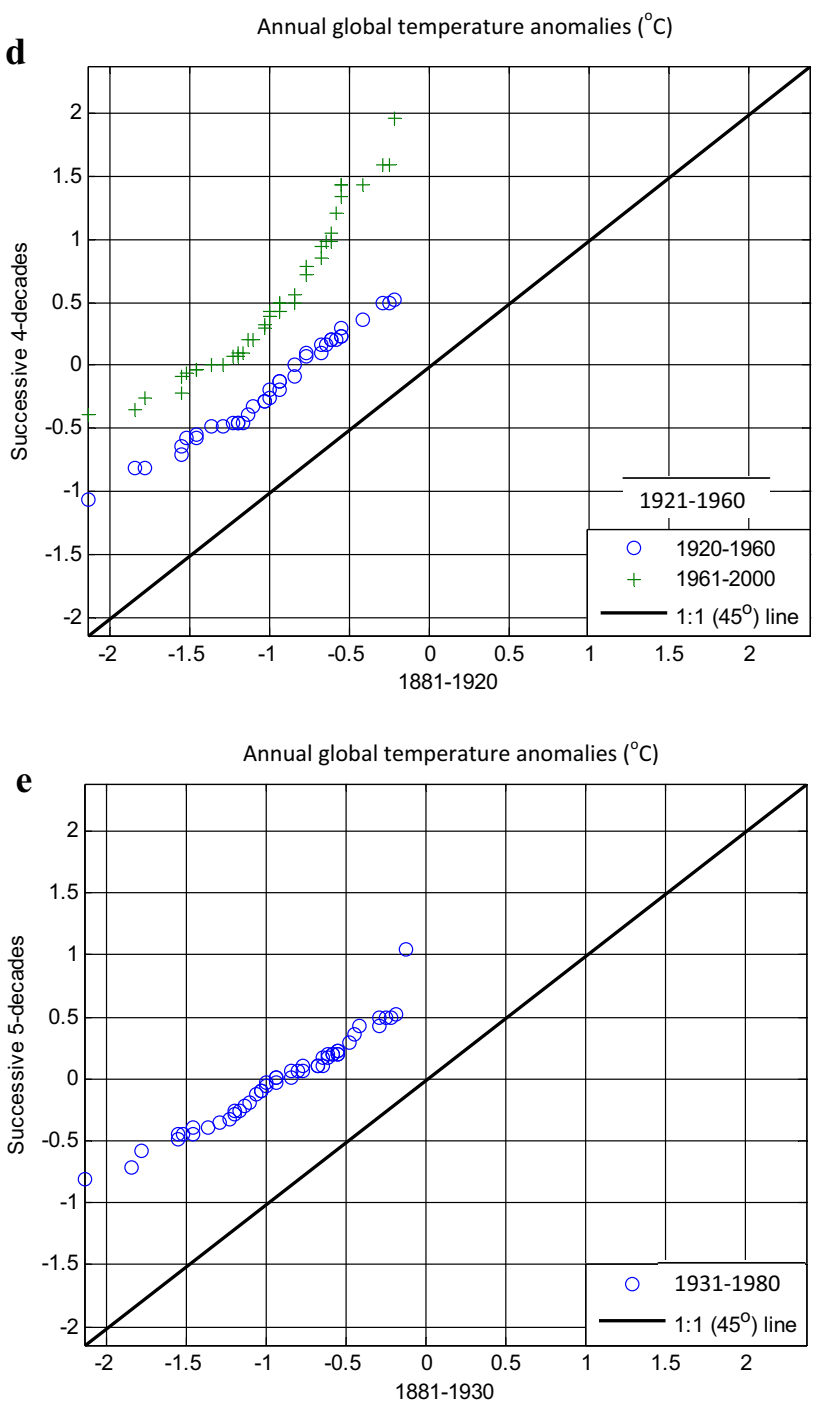

Fig. 9 continued

the 1931-1970 period where the scatter points do not fall around a parallel straight line, and hence, there are heteroscedastic (standard deviation) variations.

\subsubsection{Multi-Duration Monotonic Trend Sequence}

According to what have been explained in the previous section, it is possible to construct a sequence of monotonic trends, depending on the window width along a given time series. Furthermore, the slope, $S_{i}$, of $i$-th window is calculated by use of the following formulation (Şen, 2015):

$S_{i}=\frac{\left(m_{i 2}-m_{i 1}\right)}{n_{i}}$,

where $m_{2 i}, m_{1 i}$, and $n_{i}$ are the means of the first and second halves of time series within a window width, $n_{i}$, respectively. Another version of multi-decade ITT graphs in 
Fig. 10 Five-year multiduration trends

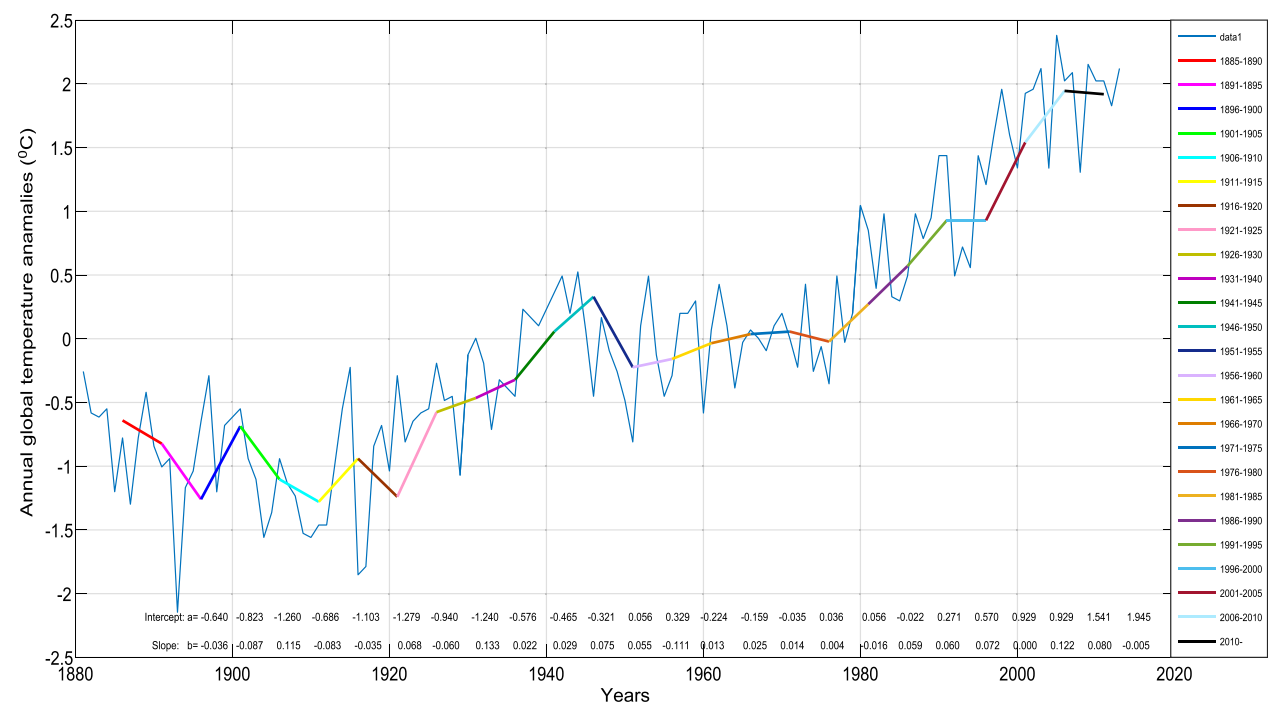

Fig. 11 Ten-year multiduration trends

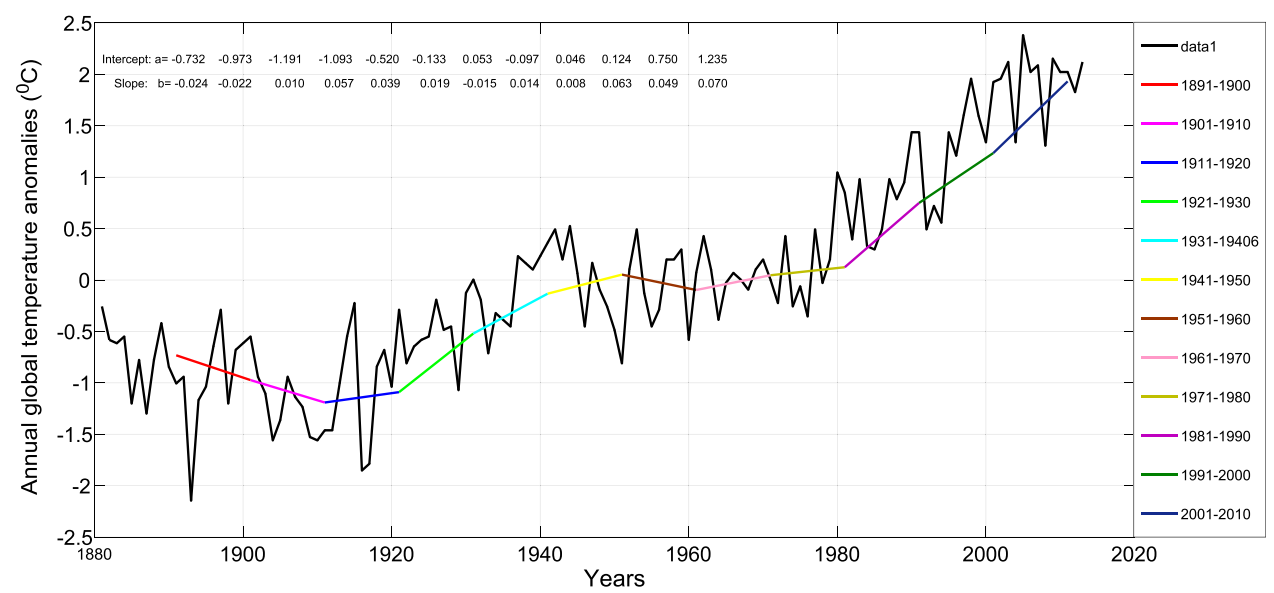

Fig. 9 is given in Figs. 10 and 11 for 5- and 10-year multiduration trends within the global temperature time series, respectively. The verbal interpretations from these figures are as follows:

1. In Fig. 4, 5-year running mean values are given without any quantitative trend component. Figure 10 provides an alternative with numerical intercept and slope values for each 5-year duration, and hence, one is able to visualize the sequence of trends and, if necessary, each trend can be quantified with a small linear mathematical expression similar to Eq. (3).

2. Each 5-year duration trend component can be related to earth system behavior during the same period, which may be due to volcanic eruptions, greenhouse gas increase, or relative decrease in the atmosphere, earthquakes, and other similar natural and anthropogenic effects.

3. Each 5-year trend component can be grouped into similarity classes according to the slope values, which also represent the intensity of warming at global scale in units of ${ }^{\circ} \mathrm{C}$ per year.
Figure 11 provides a similar multi-duration trend component sequence for each subsequent non-overlapping 10-year period, and hence, one is able to know the global change rate on decadal basis with similar interpretations to 5 -year duration.

The intercept and slope values that are obtained by the ITT analysis method on 5- and 10-year multi-duration cases are shown in Table 2.

There are 25 pieces of 5-year duration trends and the arithmetic average of this duration is 0.021 as in Table 2 . Hence, the 5-year duration temperature change can be calculated approximately as $25 \times 0.021=0.53{ }^{\circ} \mathrm{C}$, which is again rather close to $0.76{ }^{\circ} \mathrm{C}$ as suggested by IPCC (2007).

Finally, Figs. 12 and 13 represent the intercept and slope values graphical representations for 5-year period multiduration trends. It is obvious from Fig. 12 that intercept increment starts to have persistently positive values around 1970 onwards, which again corresponds to the IPCC (2007) conclusion that the climate change has started to increase steadily after 1970 . 
Table 2 Multi-duration trend intercept and slope

\begin{tabular}{|c|c|c|c|c|c|}
\hline 5-year duration & Intercept & Slope & 10-year duration & Intercept & Slope \\
\hline $1881-1885$ & & & $1981-1890$ & & \\
\hline $1886-1890$ & -0.641 & -0.036 & $1891-1900$ & -0.732 & -0.024 \\
\hline $1891-1895$ & -0.823 & -0.072 & $1901-1910$ & -0.973 & -0.029 \\
\hline $1896-1900$ & -1.260 & +0.115 & $1911-1920$ & -1.191 & +0.010 \\
\hline $1901-1905$ & -0.686 & -0.083 & $1921-1930$ & -1.090 & +0.057 \\
\hline 1906-1910 & -1.103 & -0.035 & $1931-1940$ & -0.520 & +0.039 \\
\hline $1911-1915$ & -1.279 & +0.068 & $1941-1950$ & -0.133 & +0.019 \\
\hline $1916-1920$ & -0.940 & -0.060 & $1951-1960$ & 0.053 & -0.015 \\
\hline $1921-1925$ & -1.240 & +0.133 & $1961-1970$ & -0.097 & +0.014 \\
\hline $1926-1930$ & -0.576 & +0.022 & $1071-1980$ & +0.046 & +0.008 \\
\hline $1931-1935$ & -0.465 & +0.029 & $1981-1990$ & +0.124 & +0.063 \\
\hline $1936-1940$ & -0.322 & +0.076 & $1991-2000$ & +0.750 & +0.048 \\
\hline $1941-1945$ & +0.056 & +0.055 & $2001-2010$ & +1.235 & +0.070 \\
\hline $1946-1950$ & +0.329 & -0.111 & & & \\
\hline $1951-1955$ & -0.224 & +0.013 & & & \\
\hline $1956-1960$ & -0.159 & +0.025 & & & \\
\hline $1961-1965$ & -0.035 & +0.014 & & & \\
\hline 1966-1970 & 0.036 & +0.004 & & & \\
\hline $1971-1975$ & 0.056 & -0.016 & & & \\
\hline 1976-1980 & -0.022 & +0.059 & & & \\
\hline $1981-1985$ & +0.271 & +0.060 & & & \\
\hline 1986-1990 & +0.571 & +0.072 & & & \\
\hline 1991-1995 & +0.929 & +0.000 & & & \\
\hline 1996-2000 & +0.928 & +0.122 & & & \\
\hline 2001-2005 & +1.541 & +0.081 & & & \\
\hline 2006-2010 & +1.945 & -0.005 & & & \\
\hline Mean & -0.125 & 0.021 & & -0.2107 & 0.0217 \\
\hline Standard deviation & 0.846 & 0.064 & & 0.736 & 0.034 \\
\hline
\end{tabular}

Fig. 12 Five-year duration intercept sequence

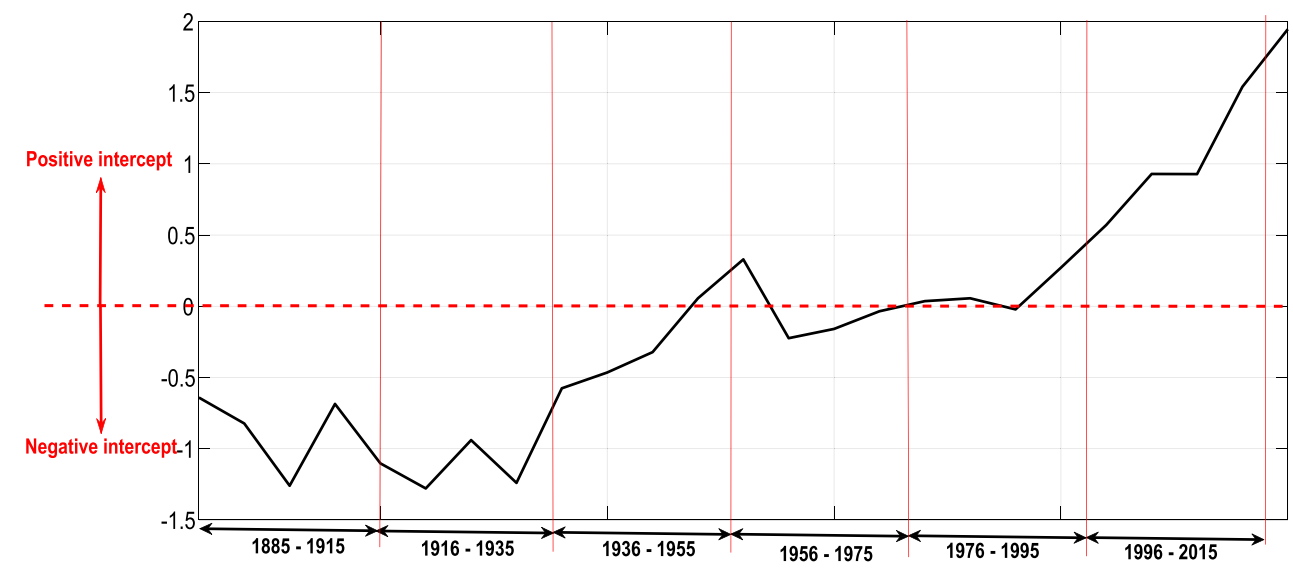

\section{Conclusions}

Climate change is one of the major problems that give rise to climate change in different regions of the Earth. The main measures of such changes are trend test analyses, which indicate the general increasing or decreasing tendencies within hydro-climatic time series record. There are two broad classical categories of trend analysis: nonparametric and parametric methodologies. Parametric regression analysis is the most preferred option, but it needs normal (Gaussian) probability distribution function (PDF) conformance of basic data, which is not possible in 
Fig. 13 Five-year duration slope sequence

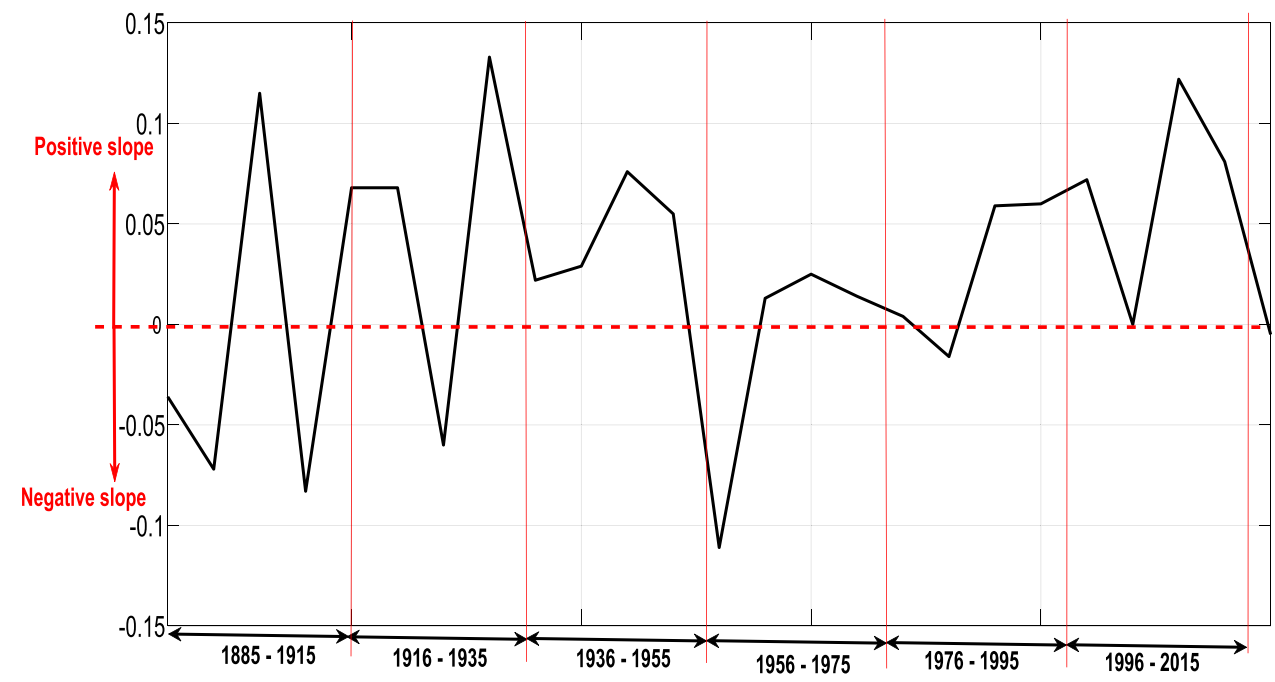

most of the hydro-climatological time series records. Nonparametric approaches destroy the natural sequence of records and depend on ascending order of the data values, and, therefore, cause loss of some information. These two categories are explained comparatively in the paper.

The innovative trend template (ITT) methodology is explained briefly and applied to global monthly temperature records from 1881 to 2013. This new methodology provides information about "low"- (minimum) and "high" (maximum)-temperature records in addition to the "Medium" transitional temperatures. First, two-half and then multi-period innovative trend analysis implementations are explained graphically, verbally, and numerically.

ITTs provide opportunity to distinguish among the "low"-, "medium"-, and "high"-temperature values in a comparative manner. In the case of two-half duration application, "low" and "high" global monthly temperature records scatter points occur along parallel lines to the 1:1 $\left(45^{\circ}\right)$ no-trend straight line, which implies that in these two categories, there are trends on the average with constant standard deviation, i.e., homoscedasticity. Through the ITTs, the warming at global scale is reached at $0.75^{\circ} \mathrm{C}$, which was determined by some other approach as $0.76 \pm 0.19{ }^{\circ} \mathrm{C}($ IPCC 2007).

Acknowledgements The authors are grateful to King Abdulaziz University for providing the computational facilities to carry out this study. Analysis of the data was performed on the Aziz Supercomputer at King Abdulaziz University's High Performance Computing Center, Jeddah, Saudi Arabia.

Open Access This article is distributed under the terms of the Creative Commons Attribution 4.0 International License (http://crea tivecommons.org/licenses/by/4.0/), which permits unrestricted use, distribution, and reproduction in any medium, provided you give appropriate credit to the original author(s) and the source, provide a link to the Creative Commons license, and indicate if changes were made.

\section{References}

Burn DH, Hag Elnur MA (2002) Detection of hydrological trends and variability. J Hydrol 255:107-122

Cailas MD, Cavadias G, Gehr R (1986) Application of a nonparametric approach for monitoring and detecting trends in water quality data of the St. Lawrence River. Water Pollut Res J Can 21:153-167

Cohn TA, Lins HF (2005) Nature's style: naturally trendy. Geophys Res Lett 32:L23402

Dabanli I, Şen Z, Yeleğen MÖ, Şişman E, Selek B, Güçlü YS (2016) Trend assessment by the innovative-şen method. Water Resour Manage. doi:10.1007/s11269-016-1478-4

Demaree GR, Nicolis C (1990) Onset of Sahelian drought viewed as a fluctuation-induced transition. Q J R Meteorol Soc 116:221-238

Gan TY (1998) Hydro-climatic trends and possible climatic warming in the Canadian prairies. Water Resour Res 34:3009-3015

Hamilton JP, Whitelaw GS, Fenech A (2001) Mean annual temperature and annual precipitation trends at Canadian biosphere reserves. Environ Monit Assess 67:239-275

Hansen J, Ruedy R, Sato M, Imhoff M, Lawrence W, Easterling D, Peterson T, Karl T (2001) A closer look at United States and global surface temperature change. J Geophys Res 106(D20):23947-23963

Hansen JW, Mason S, Sun L, Tall A (2010) Review of seasonal climate forecasting for agriculture in sub-Saharan Africa. Experimental Agriculture (in press)

Hipel KW, McLeod AI, Weiler RR (1988) Data analysis of water quality time series in Lake Erie. Water Resour Bull 24:533-544

Hirsch RM, Slack JR (1984) A nonparametric trend test for seasonal data with serial dependence. Water Resour Res 20:727-732

Hirsch RM, Slack JR, Smith RA (1982) Techniques of trend analysis for monthly water quality analysis. Water Resour Res 18:107-121

Intergovernmental Panel on Climate Change (IPCC) (2007) Climate change 2007: the physical science basis. Contribution of Working Group I to the Fourth Assessment Report of the Intergovernmental Panel on Climate Change. Cambridge Univ Press, New York

Intergovernmental Panel on Climate Change (IPCC) (2013). Edited by Thomas F. Stocker Dahe Qin, Gian-Kasper Plattner Melinda M.B. Tignor Simon K. Allen Judith Boschung, Alexander Nauels Yu Xia Vincent Bex Pauline M. Midgley and Working Group I Technical Support Unit, Working Group I Contribution 
to the Fifth Assessment Report of the Intergovernmental Panel on Climate Change (IPCC), Summary for Policymakers

Kalra A, Piechota TC, Davies R, Tootle GA (2008) Changes in U.S. streamflow and western U.S. snowpack. J Hydrol Eng 13:156-163

Kendall MG (1975) Rank correlation methods. Oxford Univ. Press, New York

Koutsoyiannis D, Montanari M (2007) Statistical analysis of hydroclimatic time series: uncertainty and insights. Water Resour Res 43(5):W05429. doi:10.1029/2006WR005592

Lins HF, Slack JR (1999) Streamflow trends in the United States. Geophys Res Lett 26:227-230

Maass A, Hufschmidt MM, Dorfman R, Thomas HA Jr, Marglin SA, Fair GM (1962) Design of water resources systems. Harvard University Press, Cambridge

Mann HB (1945) Nonparametric tests against trend. Econometrica 13:245-259

Milly PCD, Betancourt J, Falkenmark M, Hirsch RM, Kundzewicz ZW, Lettenmaier DP, Stouffer RJ (2008) Stationarity is dead: whither water management? Science 319(5863):573-574

Sen PK (1968) Estimates of the regression coefficient based on Kendall's tau. J Am Stat Assoc 63:1379-1389

Şen Z (2012) Innovative trend analysis methodology. J Hydrol Eng 17(9):1042-1046

Şen Z (2017a) Innovative trend methodologies in science and engineering. Springer, Heidelberg, Germany, p 349

Şen Z (2017b) Innovative trend significance test and applications. Theor Appl Climatol 127:939-947
Taylor CH, Loftis JC (1989) Testing for trend in lake and groundwater quality time series. Water Resour Bull 25(4):715-726

van Belle VG, Hughes JP (1984) Nonparametric tests for trends in water quality. Water Resour Res 20:127-136

Visser H, Sönke D, Petersen AC (2015) A review of trend models applied to sea level data with reference to the "accelerationdeceleration debate. J Geophys Res Oceans 120:3873-3895

von Storch H (1995) Misuses of statistical analysis in climate research. In: Storch HV, Navarra A (eds) Analysis of climate variability: applications of statistical techniques. Springer, New York, pp 11-26

Yu YS, Zou S, Whittemore D (1993) Non-parametric trend analysis of water quality data of river in Kansas. J Hydrol 150:61-80

Yue S, Wang C (2004) The Mann-Kendall test modified by effective sample size to detect trend in serially correlated hydrological series. Water Resour Managem. 18:201-218. doi:10.1023/B: WARM.0000043140.61082.60

Yue S, Pilon P, Cavadias G (2002) Power of the Mann-Kendall and Spearman's rho tests for detecting monotonic trends in hydrogeological series. J Hydrol 259:254-271

Zhang X, Harvey KD, Hogg WD, Yuzyk TR (2001) Trends in Canadian streamflow. Water Resour Res 37(4):987-998

Zhang LA, Wang MH, Hu J, Ho YS (2010) A review of published wetland research, 1991-2008: ecological engineering and ecosystem restoration. Ecol Eng 36(8):973-980 